\title{
Visual Indicator Based on Leuco Crystal Violet for Radiation Processing Technology
}

\author{
S. H. Shinde, S. Mondal, and V. Sathian
}

\begin{abstract}
One of the important technologies for food preservation and processing is radiation processing, which is growing at ever increasing rate in India. Efforts are being done to make it more cost-effective, so there is always a need for cost-effective, indigenously developed visual indicators for providing an easy identification and segregation of irradiated products. Thus development of cost-effective visual indicator based on leuco crystal violet for doses $\geq 10 \mathrm{kGy}$ was under taken. Current research works deals with fabrication and characterization of various parameters such as optimum composition, light stability, temperature effect and effect of relative humidity on the new indicator.
\end{abstract}

Keywords—Visual indicator, Radiation processing technology, Leuco crystal violet, Reflectance.

\section{INTRODUCTION}

$\mathrm{R}$ adiation processing technology is a field that makes use of large doses of radiation such as gamma rays, accelerated electrons and X-rays in-order to achieve specific biological, chemical or physical effects in a specified product [1]. In all radiation processing, accurate dosimetry ensures that the radiation treatment required for the process is correctly applied. But in-order to determine whether a product has been irradiated or not, the techniques used are generally based on physical, chemical, biological, and microbiological changes in irradiated products. However, none of these methods have the potential to effectively characterize all products in accordance to their irradiation history and usually multiple methods are need to be applied. However, a simpler method is to use self-adhesive visual indicators on product boxes. These indicators change color on irradiation to a specified dose; thus indicates visually whether or not a product has been irradiated and help in distinguishing irradiated process loads from the unirradiated process loads [2].

Availability of high intensity cobalt-60 gamma ray sources and high power electron beam accelerators has led to a continuous growth of radiation processing industry in India. There is a huge demand for indigenously developed cost-effective visual indicator. Hence an attempt was made to develop a visual indicator for high dose food radiation processing applications i.e. for doses $\geq 10 \mathrm{kGy}$. The mechanism of the radiationinduced color change of the LCV from colorless to deep purple can be attributed to formation of the highly coloured quinoid chromophore as a part of resonant carbonium cation $\left(\mathrm{CV}^{+}\right)$. Use of thin films containing leuco crystal violet (LCV) in poly vinyl butyral for high dose dosimetry in the range of $1-100 \mathrm{kGy}$ has been reported [3]. These PVB-LCV films were meant to be used as dosimeters and not as visual indicators hence light stability for short period of 3 days, was considered insufficient for visual indicator. Also, when such a system is to be proposed as a visual indicator, it should be least affected by varied environment factors such as light, temperature and humidity. This became the hypothesis for the development of visual indicator; as LCV is easily available commercially and also very cost-

S. H. Shinde, S. Mondal and V. Sathian are with Radiation Safety Systems Division, Bhabha Atomic Research Centre, Mumbai- 400085 , India. E-mail: shshinde456@gmail.com. effective as compared to diacetylenes used in some of the visual indicators [4][5][6][7], and [8].

\section{METHOD OF RESEARCH}

All reagents and solvents were obtained from Merck, Germany and used without further purification. All glasswares were cleaned as per the recommended procedure [9]. The optimum LCV concentration in the indicator required for obtaining the significantly visible color change;was determined by preparing 1, 2, 3 and 4 $\%$ solution of LCV in solvent mixture of trichloroethanol and toluene $(1 / 4 \mathrm{v} / \mathrm{v})$ containing $20 \%$ polystyrene, $1 \%$ tinuvin-327 i.e. 2-tert-Butyl-6-(5-chloro-2Hbenzotriazol-2-yl)-4-methylphenol and 1\% Irganox-1076 i.e. Octadecyl 3,5-di-tert-butyl-4-hydroxyhyrocinnamate. All the reagents were mixed thoroughly.

20 number of paper strips each having dimensions of $30 \mathrm{~mm}$ width and $60 \mathrm{~mm}$ length, were cut from a single spotless whiteA4 size paper. Length of $40 \mathrm{~mm}$ of these strips were dipped into these solutions and kept hanging for drying in dark placefor $24 \mathrm{hrs}$ and further stored at room temperature under normal laboratory conditions. This coating method produced uniformly thick indicator, each having an average thickness of $0.052 \pm 0.0035 \mathrm{~mm}$, measured by thickness gauge (Mitutoyo, Japan). Gamma Chamber-1200 was used for irradiation. It was calibrated at the centre position of its irradiation volume as per the recommended procedure [10]; using Fricke dosimeter - a reference standard [11]. Specially designed perspex jig was used for providing reproducible geometry and electronic equilibrium during irradiation of the indicators. Gamma Chamber-1200 was found to have a dose rate of $25.35 \mathrm{Gymin}^{-1}$. Irradiation temperatures encountered during irradiation were around $30^{\circ} \mathrm{C}$.

UV-Vis spectrophotometer (Shimadzu UV 3600 Plus, Japan) along with ISR-603 integrating sphere attachment was used to measure the reflectance spectra of unirradiated and irradiated in the wavelength range of 450-700 nm. Absorbance values were then calculated from these reflectance spectra assuming diffuse reflectance for these indicators irradiated to various doses. Fig. 1 represents the absorbance spectra for indicators having varied LCV concentrations irradiated to different doses. For each of the LCV concentration, indicators were irradiated in triplicates and then absorbance values were calculated. Errors bars in Fig. 1 represent the standard deviation in absorbance values. 


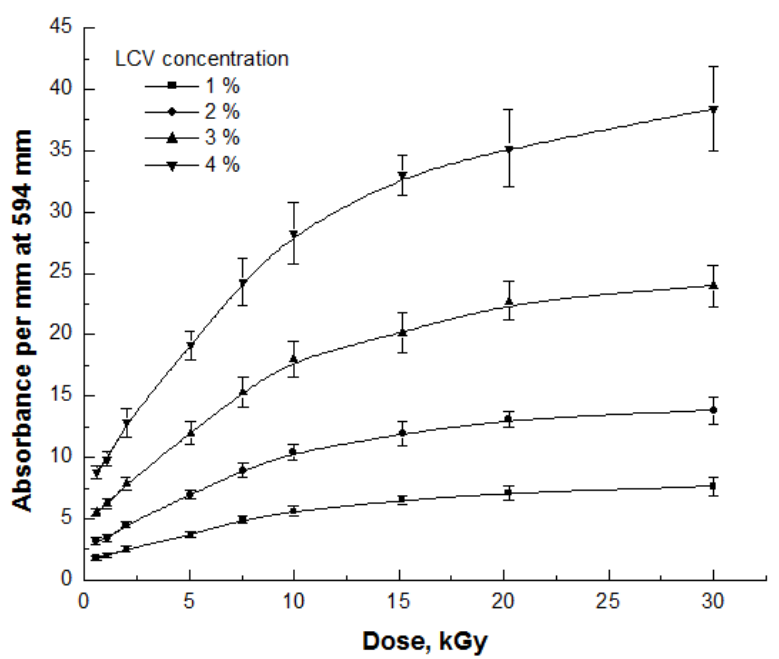

Figure 1. Change in response of irradiated labels having varied LCV concentration.

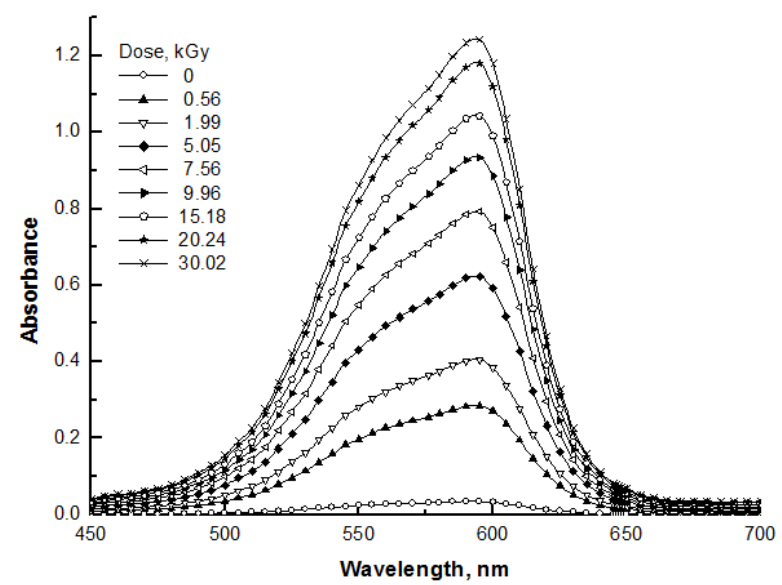

Figure 2. Absorbance spectra of unirradiated and irradiated labels.

Fig. 2 represents absorbance spectra for indicators having 3\% LCV irradiated to different doses.

Pre-irradiation and post-irradiation light stability of these indicators was studied by storing the unirradiated and irradiated indicators under normal fluorescent light along with scattered daylight in laboratory at room temperature for a period of 60 days. These indicators were not exposed to direct sunlight. Figure $3 \& 4$ shows the change in response of unirradiated and irradiated indicators respectively.

Effect of pre-irradiation storage temperature on response of the indicators was studied in the temperature range of 25 to $65^{\circ} \mathrm{C}$. Indicators were kept in a precleaned petri dish and placed inside constant temperature hot air oven (Shital Industries, India) at the required preset temperature for a period of $5 \mathrm{hrs}$ and then irradiated to a dose of $10 \mathrm{kGy}$. The reflectance values at $594 \mathrm{~nm}$ for each of these indicators were measured. Response of these indicators were normalized with respective to that at $25^{\circ} \mathrm{C}$ and are as represented as Fig. 5.

Effect of post-irradiation storage temperature on irradiated indicators was studied in the temperature range of 25 to $65^{\circ} \mathrm{C}$. Indicators were initially irradiated to a dose of $10 \mathrm{kGy}$ and kept in a pre-cleaned petri dish and placed inside constant temperature hot air oven (Shital Industries, India) at the required preset temperature for a period of $5 \mathrm{hrs}$ and then the reflectance values at $594 \mathrm{~nm}$ were measured for each of these indicators. Response of

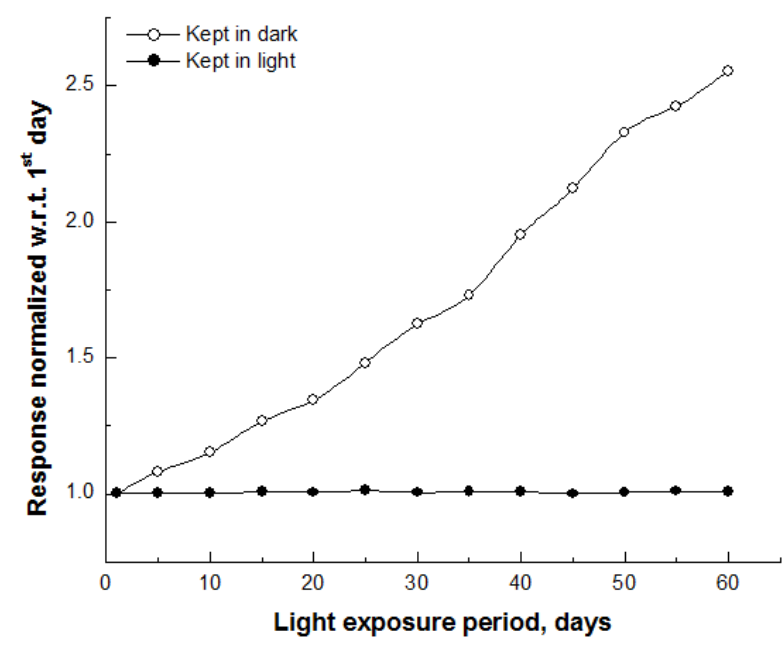

Figure 3. Light stability of unirradiated labels.

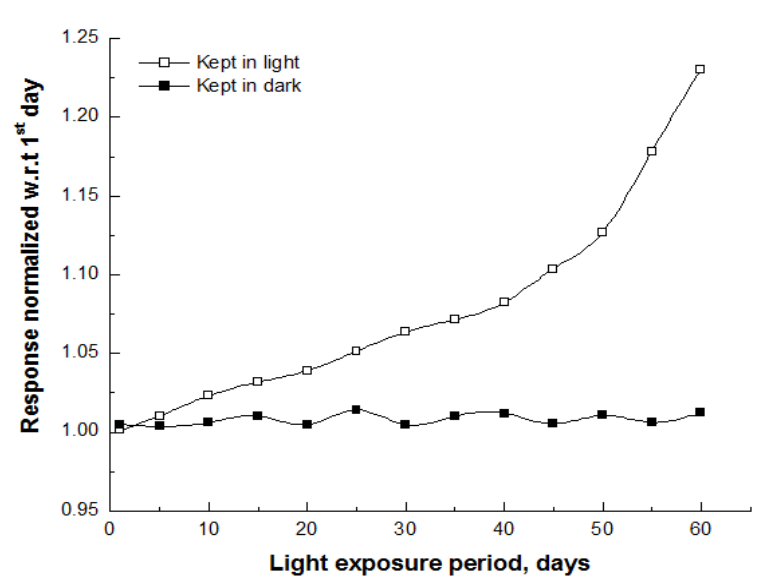

Figure 4. Light stability of irradiated labels.

these indicators were normalized with respective to that at $25^{\circ} \mathrm{C}$ and are as represented as Fig. 6.

The effect of relative humidity (RH) on the dose response of these indicators was investigated in the $\mathrm{RH}$ range from 0 to $97 \%$. Unirradiated indicators were kept in a pre-cleaned petri dish and placed inside desiccators containing the required saturated salt solution in order to establish various relative humidity ranging from 12 to 97\% [12][13]. Table 1 shows the list of salts used for this purpose. RH was measured using a digital hygrometer with an in-built temperature sensor. Zero percent RH was obtained by using dried silica gel.

Figure 7 shows the set-up for conditioning indicators to various $\mathrm{RH}$ conditions ranging from 0 to $97 \%$. For each $\mathrm{RH}$, indicators were conditioned for a period of 10 days.

All these conditioning exercises were conducted at average room temperature of $27.2{ }^{\circ} \mathrm{C}$. Each of these indicators was then sealed packed in polythene bags and irradiated to dose of $10 \mathrm{kGy}$. The reflectance values at $594 \mathrm{~nm}$ for each of these indicators were measured. Response of these indicators were normalized with respective to that at $0 \% \mathrm{RH}$ and are as represented as Figure 8.

\section{RESULT AND DISCUSSION}

The reflectance spectra of unirradiated indicators as well as irradiated indicators with doses in the range of 2.5 to $50 \mathrm{kGy}$ were measured in the visible spectrum 


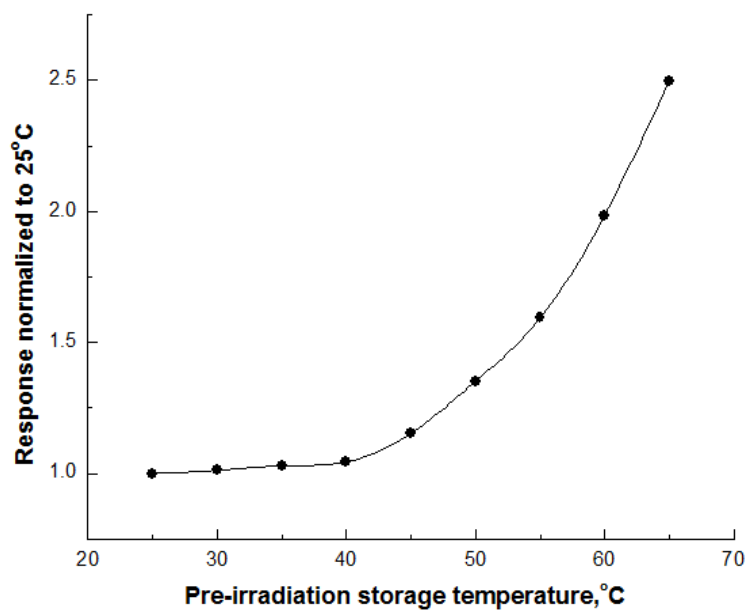

Figure 5. Change in response of labels with pre-irradiation storage temperature.

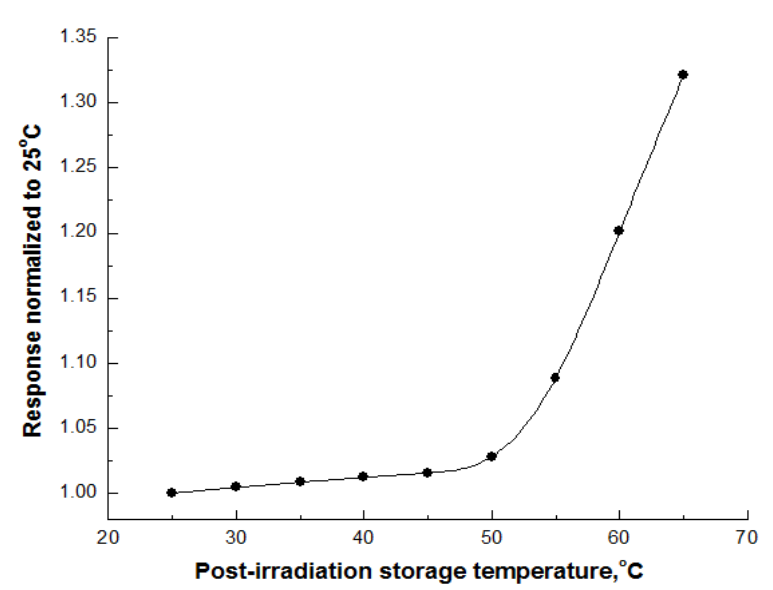

Figure 6. Change in response of labels with post-irradiation storage temperature.

range of 450-700 nm. Absorbance values were then calculated from these reflectance spectra assuming diffuse reflectance for these indicators irradiated to various doses. It is evident from the Fig. 1, that with increasing LCV concentration; the response also increases. The response for $1 \& 2 \% \mathrm{LCV}$ indicators is significantly low and for $4 \% \mathrm{LCV}$ indicators, the response increases significantly even after $10 \mathrm{kGy}$. As it was attempted to develop indicator for doses in the range of $10 \mathrm{kGy}$, optimum concentration of $3 \%$ was selected, as the response is significantly high for doses $\geq 10 \mathrm{kGy}$ and it increases steadily till $30 \mathrm{kGy}$ as compared to that for $4 \% \mathrm{LCV}$ indicators.

Figure 2 represents absorbance spectra for these indicators having 3\% LCV. It is clear from Fig. 2 that wavelength of maximum absorbance is $594 \mathrm{~nm}$. In-order to normalize the response for variations in indicator thickness, absorbance values at $594 \mathrm{~nm}$ were divided by the average indicator thickness of $0.052 \mathrm{~mm}$, response thus obtained was then plotted for respective dose values.

Effect of light during pre-irradiation and postirradiation storage of these indicators was studied by storing these indicators under normal fluorescent light along with scattered daylight in laboratory at average room temperature of $26.2{ }^{\circ} \mathrm{C}$ for a period of 60 days. At regular intervals of time, reflectance values were measured at $594 \mathrm{~nm}$ and normalized with respect to that

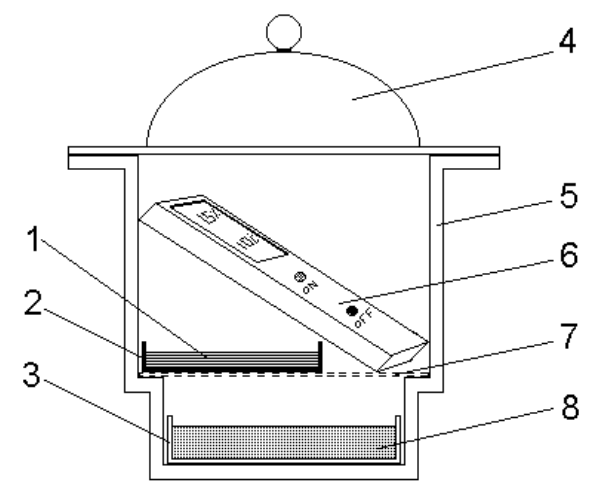

$1 \rightarrow$ Labels, $2 \rightarrow$ Petri dish (small), $3 \rightarrow$ Petri dish (large), $4 \rightarrow$ Lid, $5 \rightarrow$ Desiccator $6 \rightarrow$ Digital hygrometer, $7 \rightarrow$ Wire mesh, $8 \rightarrow$ Saturated salts

Figure 7. Set-up for conditioning labels to different RH.

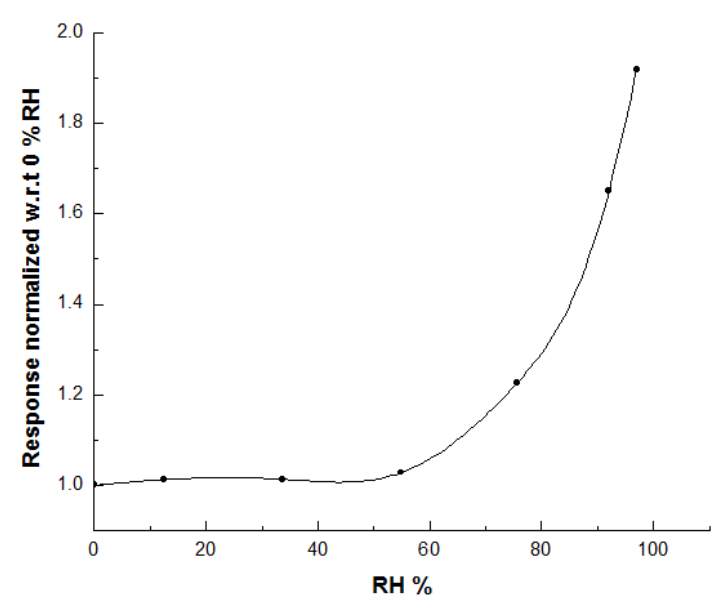

Figure 8. Change in response of labels with varied humid during pre-irradiation storage.

at $1^{\text {st }}$ day. From Fig. $3 \& 4$, it can be inferred that irradiated and unirradiated indicators stored in dark place are unaffected in terms of response during 60 days of storage period as variation in response does not exceed $1.5 \%$ and $2 \%$ over the storage period for irradiated and unirradiated indicators, respectively. But, unirradiated indicators stored under laboratory fluorescent light show significant increase in response after 40 days of storage period, however the increase of response of irradiated indicators is much slower. As the amount of leuco-form of LCV dye in unirradiated indicator is much higher than that in irradiated indicator, the unirradiated indicator is more affected by light than the irradiated indicator. During the storage period of 60 days, it was found that the increase in response for the unirradiated indicators was drastically high to about $250 \%$ and was significantly low to $22 \%$ for irradiated indicators.

Pre-irradiation and post-irradiation storage temperatures may have profound effect on the response of unirradiated and irradiated indicators respectively; hence these indicators were exposed to temperatures in the range of 25 to $65^{\circ} \mathrm{C}$ using constant temperature hot air oven. Each of the irradiated indicators was exposed to a dose of $10 \mathrm{kGy}$. Response of these indicators was normalized with respect to that at $25^{\circ} \mathrm{C}$ and plotted against respective temperatures as shown in Figs. $5 \& 6$. The response of unirradiated indicators as represented in Fig. 5; increases gradually i.e. $<5 \%$ in the temperature 
TABLE 9

SATURATED SALT SOLUTIONS REQUIRED FOR OBTAINING THE DESIRED RH IN A CLOSED CONTAINER

\begin{tabular}{cc}
\hline Saturated salt solution & Relative humidity \% \\
\hline Lithium Chloride monohydrate & 12.4 \\
Magnesium Chloride Hexahydrate & 33.6 \\
Magnesium Nitrate Hexahydrate & 54.9 \\
Sodium Chloride & 75.5 \\
Potassium Nitrate & 92.0 \\
Potassium Sulphate & 97.0 \\
\hline \hline
\end{tabular}

range of 25 to $40^{\circ} \mathrm{C}$ however after $40^{\circ} \mathrm{C}$ it increases drastically to more than $250 \%$ upto $65^{\circ} \mathrm{C}$. As seen from Fig.6, the response of irradiated indicators increases $<2.5$ $\%$ in the temperature range of 25 to $50^{\circ} \mathrm{C}$ however after $50^{\circ} \mathrm{C}$ it increases significantly to more than $30 \%$ in the temperature from 55 to $65^{\circ} \mathrm{C}$

The effect of relative humidity during pre-irradiation storage conditions on the response of unirradiated was investigated by storing these indicators at different relative humidity ranging from 0 to $97 \%$ as shown in Fig.7. These indicators were then irradiated to dose of 10kGy. Fig. 8 shows the change in response normalized with respect to that at $0 \% \mathrm{RH}$, as a function of $\mathrm{RH} \%$. The variation in response of these indicators is $<3 \%$ upto $55 \% \mathrm{RH}$ but then it increases significantly with the increase of RH\%.

\section{CONCLUSIONS}

Newly developed indicator was prepared using polystyrene instead of polyvinyl butyral (PVB) as it is cost effective than PVB and commercially easily available. Optimum concentration for LCV was determined to be $3 \%$ in solvent mixture of trichloroethanol and toluene (1/4 v/v) containing 20\% polystyrene, $1 \%$ tinuvin-327 and 1\% Irganox-1076. Trichloroethanol was added to toluene solvent to sensitize the oxidation of LCV on irradiation into crystal violet carbonium cation $(\mathrm{CV}+)$, as is reported elsewhere [14]. Tinuvin-327 was used instead of Tinuvin-P i.e. 2(2H-benzotriazol-2-yl)-p-cresol; as it is cost effective. This was added as an UV absorber to protect from fluorescent lights in the lab and sunlight in outdoor conditions. There was no protection from thermal degradation in PVB-LCV films; hence Irganox-1076 was used as an anti-oxidant to protect the indicators from thermo-oxidative degradation in outdoor conditions.

The response of these indicators was studied in the dose range of 2.5 to $50 \mathrm{kGy}$ were measured in the visible spectrum range of $450-700 \mathrm{~nm}$. Wavelength of maximum absorbance was found to be $594 \mathrm{~nm}$. Effect of light during pre-irradiation and post-irradiation storage of these indicators was studied under normal laboratory fluorescent light along with scattered daylight for a period of 60 days. Irradiated and unirradiated indicators stored in dark place are unaffected in terms of response during 60 days of storage period as variation in response does not exceed $2 \%$. But, unirradiated indicators stored under laboratory fluorescent light show significant increase in response after 40 days of storage period, however the increase of response of irradiated indicators is much slower. It therefore is recommended to store unirradiated indicators in dark condition.
Effect of pre-irradiation and post-irradiation storage temperatures on the response of unirradiated and irradiated indicators was studied for temperatures in the range of 25 to $65^{\circ} \mathrm{C}$. The response variation of unirradiated indicators is $<5 \%$ upto $40^{\circ} \mathrm{C}$ however then it increases drastically upto $65^{\circ} \mathrm{C}$. Therefore, it is recommended to store unirradiated indicators under $40^{\circ} \mathrm{C}$. However, variation in response of irradiated indicators is $<2.5 \%$ upto $50^{\circ} \mathrm{C}$ and then it increases significantly upto $65^{\circ} \mathrm{C}$. Hence it is recommended to use these indicators in the temperatures $<50^{\circ} \mathrm{C}$.

As the variation in response is significantly high, when stored in relative humidity more than $55 \%$, but the variation was insignificant $(<3 \%)$ when stored in $0-55 \%$ humidity range, it is recommended to pack and seal unirradiated indicators under RH ranging from $0-55 \%$.

Thus, the newly developed indicator is having better light stability, thermal stability and more resistance to relative humidity as compared to the PVB-LCV film. Since indicators are used as qualitative rather than a quantitative tool, hence estimation of uncertainty involved in dose estimation is not relevant. Overall, this indicator has good potential to be used as indigenously developed cost-effective visual indicator for various food irradiation facilities.

\section{REFERENCES}

W. L. McLaughlin, A. W. Boyd, K. H. Chadwick, J. C. McDonald, and A. Miller, "Radiation Processing and dosimetry," in Dosimetry for Radiation Processing, 1989, pp. $1-27$.

ASTM International, "Standard Guide for Use of RadiationSensitive Indicators ISO/ASTM 51539," West Conshohocken, 2013.

Y. Soliman and A. Abdel-Fattah, "Leuco crystal violet / poly (vinyl butyral) thin film as a high-dose dosimeter," Radiat. Meas, vol. 49, pp. 1-6, 2013.

G. Patel, "Diacetylenes as Radiation Dosage Indicators," Radiat. Phy. Chem, vol. 18, pp. 913-925, 1981.

A. Abdel-Fattah, Y. Soliman, A. Bayomi, and A. AbdelKhalek, "Dosimetric characteristics of a Radiochromic polyvinyl butyral film containing 2,4-hexadiyn-1,6-bis(nbutyl urethane)," Appl. Radiat. Isot, vol. 86, pp. 21-27, 2014. T. Alkhuraiji, Y. Soliman, and A. Abdel-Fattah, "Radiochromic film containing ploy (hexa-2,4-diynylene adipate) as a radiation dosimeter," Appl. Radiat. Isot, vol. 141, pp. 80-87, 2018.

Y. Soliman, A. Bayomi, A. Abdel-Fattah, and A. AbdelKhalek, "Radiochromic label dosimeter based on a synthesized monomer of 2,4-hexadiyn-1,6-bis(n-butyl urethane)," Sens. Actu. B Chem, vol. 200, pp. 109-116, 2014. T. Prusik, M. Montesalvo, and T. Wallace, "Use of Polydiacetylenes in an Automated Label Dosimetry System," Radiat. Phy. Chem., vol. 31, no. 2, pp. 441-447, 1997.

B. Gupta, R. Bhat, G. Narayan, and S. R. Nilekani, "A spectrophotometric readout method for free radical dosimetry," Radiat. Phy. Chem., vol. 26, pp. 647-656, 1985. ASTM International, "Standard Practice for Dosimetry for a Self-Contained Dry-Storage Gamma Irradiator," West Conshohocken, 2013.

A. International, "Practice for using the Fricke dosimetry system ISO/ASTM 51026," West Conshohocken, 2015.

S. Shinde, D. Babu, and T. Mukherjee, "Effect of Relative Humidity on Dose Response of Effervescent Glycine Pellet Dosimeters," J. Sci. Tech., vol. 5, no. 1, pp. 33-48, 2013.

L. Greenspan, "Equilibrium relative humidity of some saturated salt solutions $25 \mathrm{oC}$," J. Res. Nat, vol. 81A, p. 89, 1977.

T. Miyaji, S. Tokita, T. Tachikawa, and C. Azuma, "Development of a radiation dosimeter consisting of leuco crystal violet and a small amount of halocarbons," J. Phot. Sci. Tech, vol. 14, pp. 225-226, 2001. 